\title{
Children with Down Syndrome (DS), and Autism Spectrum Disorder (ASD): Difficulties of Screening and Management of This Dual Diagnosis about 3 Cases
}

\author{
Khaoula Mammad1*, Meryem Chkirat1, Yamna Kriouile², Asmaa Mdaghri Alaoui2,3* (D) \\ ${ }^{1}$ Unit of Clinical Neuroscience and Applied Nutrition, Department of Biology, Faculty of Sciences, University Ibn Tofail, Kenitra, Morocco \\ ${ }^{2}$ Pediatric Service 2, Children's Hospital, University Hospital Center (UHC), Ibn Sina, Rabat, Morocco \\ ${ }^{3}$ Congenital Anomalies Research Team, Faculty of Medicine and Pharmacy, Mohammed V University, Rabat, Morocco \\ Email: ^Khaoula.mammad@gmail.com, *amdalaoui@yahoo.fr
}

How to cite this paper: Mammad, K., Chkirat, M., Kriouile, Y., \& Mdaghri Alaoui, A. (2019). Children with Down Syndrome (DS), and Autism Spectrum Disorder (ASD): Difficulties of Screening and Management of This Dual Diagnosis about 3 Cases. Psychology, 10, 931-939. https://doi.org/10.4236/psych.2019.106060

Received: April 4, 2019

Accepted: May 27, 2019

Published: May 30, 2019

Copyright $\odot 2019$ by author(s) and Scientific Research Publishing Inc. This work is licensed under the Creative Commons Attribution International License (CC BY 4.0).

http://creativecommons.org/licenses/by/4.0/

\begin{abstract}
Down syndrome and autism spectrum disorder can be combined at the child attained; this dual diagnosis is stressful for the parents and is often overlooked by the health and education professionals of these patients. Through three observations collected at the consultation of dysmorphology of the Children's Hospital of Rabat (CHR), this article is illustrated with the details of this association, the clarification of the data of this double diagnosis and the recommendations of good practices for the screening and early management of ASD in this population of children with special needs. The $1^{\text {st }}$ case: A boy, born on 02/06/2014, unique of his family, is followed for DS. The diagnosis of DS was made at birth and confirmed by the karyotype; para-clinical assessment revealed congenital hypothyroidism $(\mathrm{CH})$ treated with thyroid hormones and without identification of other congenital anomalies; the $2^{\text {nd }}$ case: A boy, the elder of a sibling of 4 , was born on 22/12/2010, the diagnosis of DS was retained from birth, the balance did not objectify congenital pathology; the $3^{\text {rd }}$ case: A girl was born on 03/12/2009, consanguineous relative, hypotonic, eupneic, eutrophic; a mal-formative assessment on a trisomy 47, $\mathrm{XX},+21$ karyotype was realized. Diagnosis with PEP3 and CARS shows severe autistic spectrum disorder for all three patients. In the light of these three observations and of the medical literature, the authors stress the importance of screening for precursor signs in the first year of life for early stimulation by parents and neuropsycho-educational rehabilitation, in order to ensure adequate better development of brain plasticity and thus reduce parental stress and complications related to this dual diagnosis.
\end{abstract}

*Pr. Asmaa MDAGHRI ALAOUI and DrKhaoula MAMMAD were equally involved in writing the article. 


\section{Keywords}

Down Syndrome, Autism Spectrum Disorder, Early Screening, Diagnosis, CARS, PEP3

\section{Introduction}

DS is often associated with other malformation pathologies such as congenital heart disease, and endocrine: hypothyroidism and also psychiatric diseases: OCD, ADHD, and ASD. It is still often considered that the inappropriate behaviors of some people with DS are due to their intellectual and/or sensory impairments, and this population is also likely to have an Autism Spectrum Disorder (ASD) (Patterson, 1999). More recent research, however, points to a specific set of social impairments and related behavioral challenges in individuals with DS, though these have been understudied (Warner et al., 2014). For some individuals with DS, a pattern of atypical social behaviors, along with known cognitive and linguistic impairments (DiGuiseppi et al., 2010), could look similar to symptoms of autism spectrum disorder (ASD).

Biological bases for co-occurring ASD and DS have been shown that a centrosome has been implicated in numerous cellular processes that lead to various neurological diseases associated with brain structural abnormalities, through neuronal migration pathways and microtubular organization (Badano et al., 2005; Bond et al., 2006). A study through linkage analysis identified a $2.7 \mathrm{Mb}$ region on 21q among 34 autism-affected relative pairs selected for language regression. This region includes genes with known or possible roles in cellular differentiation, apoptosis, virus infection susceptibility, and responses to oxidation-reduction changes, all of which have been potentially implicated in autism [6]. However, in a different ASD population, no linkage to chromosome 21 was identified (Parr et al., 2006), which leads to conclude that the role of joint biological mechanisms in these two disorders requires further investigation.

Recent studies have reported variable rates of autism in children with Down syndrome; likely due to different diagnostic measures and different samples used in the studies some studies estimate that this joint diagnosis concerns about $1 \%$ to 19\% of people with SD (Ghaziuddin et al., 1992; Moss et al., 2012). Other studies report that somewhere between 2\% (Collacott et al., 1992), 5\% (Ghaziuddin et al., 1992), 7\% (Kent et al., 1999) and 10\% (Paly \& Hurley, 2002) of individuals with Down syndrome meet the criteria for autism or autism spectrum disorders. Others suggest that about $16 \%$ to $19 \%$ of children are with Down syndrome and $8 \%$ to $9 \%$ of them have autism, although some report higher rates (Warner et al., 2014).

This association remains a difficult situation for the patient and his family, especially before retaining this joint diagnosis by health professionals, and poses a diagnosis problem especially for professionals with little experience in very distinctive behavioral and developmental disorders. Methods of assessing autism 
symptoms in persons with Down syndrome have been limited to the use of a screening tool (Kent et al., 1999). Some studies (Capone et al., 2005; Carter et al., 2007) and Molloy et al. (2009) reported that people with ASD and DS had a higher degree of Intellectual Disability (ID) than those with only SD and who did not meet the ASD criteria. This dual diagnosis, although common, is often overlooked by health professionals caring for children with DS, which delays the appropriate management of ASD.

Through three observations collected at the dysmorphology consultation of the CHR, the objective of this article is to illustrate the particularities of this association, to specify the clinical characteristics of children with this dual diagnosis, and to establish good practice guidelines for screening and early management of ASD in this population of children with DS.

\section{Materials and Methods}

\section{Participants:}

3 children diagnosed with Down syndrome with ASD.

\section{Consent}

Approval for this study was granted by the of the children's hospital of Rabat Committee. Written informed consent was obtained from the parents or legal guardians of the 3 children participating in this study.

\section{$\underline{1^{\text {st }} \text { Clinical Case: }}$}

A boy, born on $02 / 06 / 2014$ who is unique to his family, is followed for DS, a mother born in 28/02/1996 and a father born in 08/08/1986. Pregnancy was followed. The diagnosis of SD was evoked at birth and confirmed by the karyotype $(47, \mathrm{XY},+21)$; At the trace level of EEG monitoring is normal at $02 / 07 / 214$, after the $06 / 06 / 2014$ trace of wakefulness, and sleep were normal the malformation assessment did not objectify of congenital cardiopathy nor of another malformation of $\mathrm{CH}$ was detected and the child was put on thyroid hormone supplementation, with cervical ultrasound without abnormality (see Figure 1).

The evolution was marked by a delay of the psychomotor acquisitions (holding of the head at 8 months, sitting position at 17 months, and walking alone at 4 years) and impaired language. According to Child Psychiatrist at 26/04/2018 diagnose as much as autistic

\section{$2^{\text {nd }}$ Clinical Case:}

A boy who is the eldest of a sibship of 4 . Born on December 22, 2010, a primipara

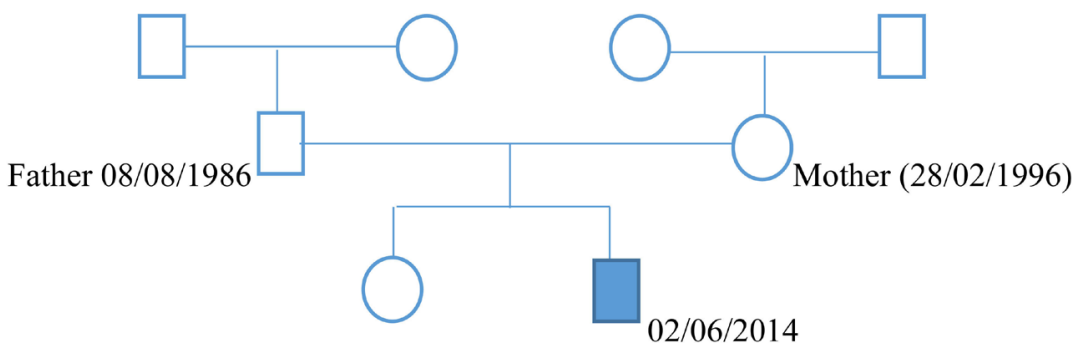

Figure 1. Family tree to $1^{\text {st }}$ clinical case. 
mother born on 28/12/1982 the diagnosis of DS was suspected from birth, the balance did not objectified nor of $\mathrm{CH}$, nor of congenital cardiopathy, the follow-up of the patient objectified a delay of the psychomotor acquisitions: sitting position at 18 months walking alone at 2 years, He said some syllables like "Ma", "Ba"; but after had a regression of the speech, with stereotyped movements with isolation (see Figure 2).

$3^{\text {rd }}$ Clinical Case:

A girl born on 03/12/2009 of a mother born in 1992, consanguineous relative, having anal imperforation for which she was operated at birth, the diagnosis of DS was evoked at birth and confirmed by the karyotype $(47, \mathrm{XX},+21)$, the malformation report also objectified an interventricular communication with the echocardiography. The psychomotor development was marked by the delay of the motor acquisitions and the appearance of a bilateral nystagmus with delay of the language. In addition, the thyroid, echo-abdominal and auditory evoked potentials were normal. Otherwise, we had an appearance of behavioral disorders with isolation at the age of 4 years (see Figure 3 ).

\section{Methods}

\section{Psychoeducational profile $3^{\text {rd }}$ edition: PEP-3}

PEP-3 was designed to help parents and educators plan educational management and diagnose autism or other pervasive developmental disorders. It consists of two parts. The Performance Scale uses direct assessment and observation of the child. It consists of 10 subtests, 6 that assess developmental abilities and 4 others that measure inappropriate behaviors. These subtests are grouped together to form 3 categories: communication, motor skills and unsuitable behaviors. In the Educator's Report, the parent or educator records their observations.

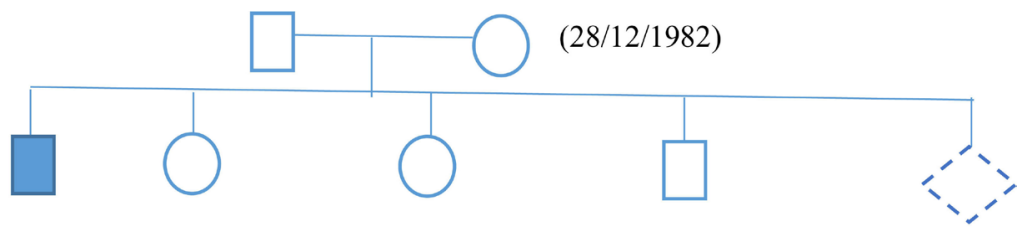

$(22 / 12 / 2010)(26 / 01 / 2012)$

$(19 / 10 / 2014)$

$(12 / 03 / 2017)$

Figure 2 . Family tree to $2^{\text {nd }}$ clinical case.

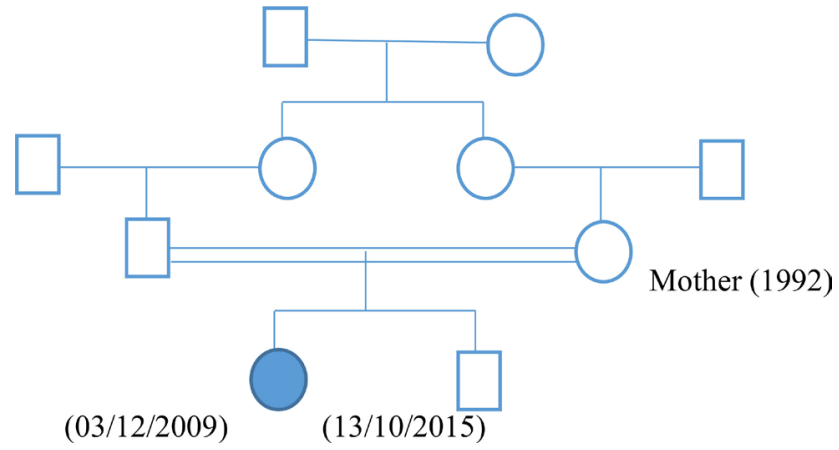

Figure 3. Family tree to $3^{\text {th }}$ clinical case. 
The Educator's Report consists of two clinical sections and three subtests. One of these subtests measures developmental skills and the other two assess maladaptive behaviors (Schopler et al., 2005).

\section{The Childhood Autism Rating Scale CARS:}

The Childhood Autism Rating Scale is a behavioral rating scale used to assess the presence and severity of autism spectrum disorder symptoms. The evaluators completed the CARS questionnaire after the developmental evaluation. Information from clinical observations, test measurements and parents' reports were used to assign CARS ratings (Schopler et al., 1980; Schopler et al., 1988).

\section{Results and Discussions}

According to the two scales used PEP3 and CARS to retain the diagnosis of ASD in the 3 patients with DS (see Table 1), we found for:

- The $1^{\text {st }}$ child: the existence of autism with a severe degree and a mental age of less than 12 months;

- The second case: severe autism with a mental age of 6 months and a physical age of 98 months;

- The $3^{\text {rd }}$ patient: severe autism with a physical age of 110 months and a mental age of 6 months.

According to our result, question regarding the role of intellectual disability (ID) in the association between genetic syndromes and ASD. Identification of ASD characteristics in individuals with severe and profound levels of ID and those with complex behavioural and cognitive profiles is particularly difficult (Moss \& Howlin, 2009).

According to the DMS V, autistic disorders are defined as one of the neurodevelopmental disorders. The updated diagnostic criteria are defined in two symptomatic dimensions which are: Persistent deficits of communication and social interactions observed in various contexts; the restricted and repetitive nature of behaviors, interests or activities (APA, 2013).

This dimensional definition is supplemented by a level of severity depending on the level of assistance required. The diagnosis of ASD is based on symptoms in three areas: social impairment, communication disorders and repetitive behaviors. Furthermore, the symptoms of autism that have been noted in studies of people with Down syndrome include: social isolation, misuse of the gaze, restricted/idiosyncratic interests, concern with parts of objects, hand flapping, body sway, compulsiveness, and misunderstanding (Ghaziuddin et al., 1992;

Table 1. Interpretation of PEP3 and CARS's scoring.

\begin{tabular}{ccccccr}
\hline & chronic age & Communication & Motricity & $\begin{array}{c}\text { Maladaptive } \\
\text { Behaviors }\end{array}$ & $\begin{array}{c}\text { Age of } \\
\text { developments }\end{array}$ & CARS \\
\hline Case 1 & 56 month & Severe & Severe & Severe & $<12$ month & severe \\
Case 2 & 98 month & Severe & Severe & Severe & 6 month & severe \\
Case 3 & 110 month & Severe & Severe & Severe & 6 month & severe \\
\hline
\end{tabular}


Kent et al., 1999).

Among the behaviors found in one of the patients was bruxism, a symptom not considered early as stereotypical behaviour until making the diagnosis after. Many of the core diagnostic features of ASD are developmentally weighted and consequently an individual may meet certain diagnostic criteria because they have not yet reached the developmental level required for that behaviour to be achieved.

Moreover, the clinical tools that have been developed to aid the diagnosis of ASD are generally not designed to be able to distinguish between these very subtle differences and complex patterns of behaviour and thus may have more limited sensitivity and specificity when applied to individuals with genetic syndromes associated with ID (Moss et al., 2011).

However, the diagnosis of ASD should only be made by experienced clinicians using standardized assessments. The diagnosis is more difficult to establish in children with DS with severe intellectual disability. There is a significant risk of false negatives, i.e. a diagnosis of delayed autism (Hepburn et al., 2007).

Among the etiological hypotheses, some authors argue in favor of a genetic etiology involving chromosome 21 and genes in the coexistence of these two pathologies. Some pathogenic mechanisms may also cause these disorders such as family history of autism, infantile spasm, epilepsy, brain damage following cardiac surgery, severe infections, hearing and visual deficits, or premature hypothyroidism, increased to develop an autism spectrum disorder (Krieger et al., 2014).

The manifestations of the disorder vary widely according to the stage of mental development and the chronological age of the subject. They begin before the age of three. In some cases, parents say they began to worry about their baby from birth or soon after, due to lack of interest in social interactions. Further analysis indicated that individuals with ASD/DS had lower levels of self-help skills, were less mobile and had fewer verbal abilities than the total sample of individuals with DS. These findings are consistent with reports from (Capone et al.,2005; Carter et al., 2007; Molloy et al., 2009; Hepburn et al., 2008).

The impact of developmental level on symptoms has also not been sufficiently explored (Howlin et al., 1995). In order to understand how autism symptoms manifest themselves in some children with Down syndrome, it is important to include a group of well-characterized children with Down syndrome and use advanced diagnostic tools to detect the symptoms of autism. Since the symptoms of autism occur before the age of 3 in classical autism, it is also essential to evaluate children with Down syndrome by their third birthday. Examining the relationship between the symptoms of autism and other characteristics of the child (level of development, temperament, for example) will be important to better understand people at risk for comorbid diagnosis, which has consequences for practice.

Some studies with Down syndrome and autism suggest that they see a number of children whose early development does not give any cause for concern but 
that they seem to "regress" and lose some skills such as some early words or signs and then progress seems to stop and a serious autistic profile emerges (Capone, 2002).

However, the retrospective clinical analysis of certain attitudes and the observation of children's family films with ASD highlight signs of alert that can be established in the first year of life or even before 6 months, namely: Early disorders of interaction: baby wise, never crying, baby "who is forgotten"; Tone disorders: In the form of hypotonia; or conversely, hypertonic reactions with ceaseless gesticulation, inability to calm down in the arms; The frequent stall of the gaze: avoidance of the gaze; strabismus is common; No voluntary smile from the 3rd month; Sleep disorders with insomnia: Calm baby, eyes wide open waiting in bed; Eating Disorders: Refusal to breastfeed, anorexia; Serious air with a mechanical relational style; An apparent indifference to separations and reunions; With often a lack of reaction to the foreigner is observed.

Children with Down syndrome are at increased risk for sensory conditions (e.g., hearing loss) and motor difficulties (e.g., hypotonia), which may affect the timing and fluidity of their social and communicative behaviors in a manner detected by screening tools, but qualitatively different from the core social relatedness problems of autism. False positive screening results were more likely in children with hearing or persistent vision problems (Rowe et al., 2006). The symptoms conventionally observed during autism are difficult to evaluate in children with DS because they can be masked by the psychomotor retardation of the chromosomal aberration itself. This explains the diagnostic delay observed in this respect (Kent et al., 1999; Capone et al., 2005; Krieger et al., 2014; Barbaresi et al., 2006). Indeed, the average age of diagnosis of autism in a child with DS is 14.4 years. Hence the importance of informing health professionals about the combination of SD and ASD for early diagnosis and therefore appropriate, multidisciplinary management including: pediatrician, neuropediatrician, child psychiatrist, speech therapist and psychiatrist, neuropsychologist (Krieger et al., 2014).

\section{Conclusion}

The co-occurrence of autism spectrum disorder (ASD) and Down syndrome has important implications for intervention. This study is limited by the small sample but we are involved in developing more research on that topic in the future. These children will likely need different treatments and educational approaches. In the light of these three observations and the medical literature, the authors point out the interest of screening for precursor signs in the first year of life for early stimulation by parents and neuropsycho-educational rehabilitation to ensure a better development of brain plasticity and thus reduce parental stress and complications related to this double diagnosis.

\section{Conflicts of Interest}

The authors declare no conflicts of interest regarding the publication of this paper. 


\section{References}

American Psychiatric Association (APA) (2013) DSM-V Diagnostic and Statistical Manual of Mental Disorders (5th ed.). Washington DC: American Psychiatric Association. https://doi.org/10.1176/appi.books.9780890425596

Badano, J. L., Teslovich, T. M., \& Katsanis, N. (2005). The Centrosome in Human Genetic Disease. Nature Reviews Genetics, 6, 194-205. https://doi.org/10.1038/nrg1557

Barbaresi, W. J., Katusic, S. K., \& Voigt, R. G. (2006). Autism: A Review of the State of the Science for Pediatrics Primary Health Care Clinicians. Archives of Pediatrics \& Adolescent Medicine, 160, 1167-1175. https://doi.org/10.1001/archpedi.160.11.1167

Bond, J., \& Woods, C. G. (2006). Cytoskeletal Genes Regulating Brain Size. Current Opinion in Cell Biology, 18, 95-101. https://doi.org/10.1016/j.ceb.2005.11.004

Capone, G. T. (2002). Down Syndrome and Autistic Spectrum Disorders. In W. I. Cohen, L. Nadel, \& M. Madnick (Eds.), Down Syndrome: Visions for the 21st Century (ch 24 pp. 327-336). New York: Wiley-Liss. https://doi.org/10.1002/0471227579.ch24

Capone, G. T., Grados, M. A., Kaufmann, W. E., Bernad-Ripoll, S., \& Jewell, A. (2005). Down Syndrome and Comorbid Autism-Spectrum Disorder: Characterization Using the Aberrant Behavior Checklist. American Journal of Medical Genetics, 134, 373-380. https://doi.org/10.1002/ajmg.a.30622

Carter, J. C., Capone, G. T., Gray, R. M., Cox, C. S., \& Kaufmann, W. E. (2007). Autistic-Spectrum Disorders in Down Syndrome: Further Delineation and Distinction from Other Behavioral Abnormalities. American Journal of Medical Genetics Part B: Neuropsychiatric Genetics, 1, 87-94. https://doi.org/10.1002/ajmg.b.30407

Collacott, R. A., Cooper, S. A., \& McGrother, A. (1992). Differential Rates of Psychiatric Disorders in Adults with Down's Syndrome Compared to Other Mentally Handicapped Adults. British Journal of Psychiatry, 161, 671-674.

https://doi.org/10.1192/bjp.161.5.671

DiGuiseppi, C., Hepburn, S., Davis, J., Fidler, D. et al. (2010) Screening for ASD in Children with Down Syndrome: Population Prevalence and Screening Test Characteristics. Journal of Developmental \& Behavioral Pediatrics, 31, 181-191.

https://doi.org/10.1097/DBP.0b013e3181d5aa6d

Ghaziuddin, M., Tsai, L. Y., \& Ghaziuddin, N. (1992). Autism in Down's Syndrome: Presentation and Diagnosis. Journal of Intellectual Disability Research, 36, 449-456. https://doi.org/10.1111/j.1365-2788.1992.tb00563.x

Hepburn, S., Philofsky, A., Fidler, D. J., \& Rogers (2008). Autism Symptoms in Toddlers with Down Syndrome: A Descriptive Study. Journal of Applied Research in Intellectual Disabilities, 21, 48-57.

Hepburn, S., Philofsky, A., Fidler, D., \& Rogers, S. (2007). Autism Symptoms in Toddlers with Down Syndrome: A Descriptive Study. Journal of Applied Research in Intellectual Disabilities, 21, 48-57. https://doi.org/10.1111/j.1468-3148.2007.00368.x

Howlin, P., Wing, L., \& Gould, J. (1995). The Recognition of Autism in Children with Down syndrome: Implications for Intervention and Some Speculations about Pathology. Developmental Medicine and Child Neurology, 37, 406-414. https://doi.org/10.1111/j.1469-8749.1995.tb12024.x

Kent, L., Evans, J., Paul, M., \& Sharp, M. (1999). Comorbidity of Autistic Spectrum Disorders in Children with Down Syndrome. Developmental Medicine and Child Neurology, 41, 153-158. https://doi.org/10.1017/S001216229900033X

Krieger, A.-E. et al. (2014). Trisomie 21 et autisme: Double diagnostic, évaluation et intervention. Neuropsychiatrie de l'enfance et de l'adolescence, 62, 235-243. 
https://doi.org/10.1016/j.neurenf.2014.02.006

Molloy, C. A., Murray, D. S., Kinsman, A., Castillo, H., Mitchell, T., Hickey, F. J., \& Patterson, B. (2009). Differences in the Clinical Presentation of Trisomy 21 with and without Autism. Journal of Intellectual Disability Research, 53, 143-151. https://doi.org/10.1111/j.1365-2788.2008.01138.x

Moss, J., \& Howlin, P. (2009). Invited Annotation-Autism Spectrum Disorders in Genetic Syndromes: Implications for Diagnosis, Intervention and Understanding the Wider ASD Population. Journal of Intellectual Disability Research, 53, 852-872. https://doi.org/10.1111/j.1365-2788.2009.01197.x

Moss, J., Howlin, P., \& Oliver, C. (2011). The Assessment and Presentation of Autism Spectrum Disorder and Associated Characteristics in Individuals with Severe Intellectual Disability and Genetic Syndromes. In J. Burack, R. Hodapp, G. Iarocci, \& E. Zigler (Eds.), The Oxford Handbook of Intellectual Disability and Development (pp. 1-57). New York: Oxford University Press. https://doi.org/10.1093/oxfordhb/9780195305012.013.0018

Moss, J., Richards, C., Nelson, L., \& Oliver, C. (2012). Prevalence of Autism Spectrum Disorder Symptomatology and Related Behavioural Characteristics in Individuals with Down Syndrome. Autism, 17, 390-404. https://doi.org/10.1177/1362361312442790

Paly, R. J., \& Hurley, A. D. (2002). Down Syndrome and Autistic Disorder. Mental Health Aspects of Developmental Disabilities, 5, 64-65.

Parr, J. R., Lamb, J. A., Bailey, A. J., \& Monaco, A. P. (2006). Response to Paper by Molloy et al.: Linkage on 21q and 7q in Autism Subset with Regression. Molecular Psychiatry, 11, 617-619. https://doi.org/10.1038/sj.mp.4001833

Patterson, B. (1999). Dual Diagnosis: The Importance of Diagnosis and Treatment. Disability Solutions, 3, 16-17.

Rowe, J., Lavender, A., \& Turk, V. (2006). Cognitive Executive Function in Down's Syndrome. British Journal of Clinical Psychology, 45, 5-17. https://doi.org/10.1348/014466505X29594

Schopler, E., Lansing, M. D., Reichler, R. J., \& Marcus, L. M. (2005). Examiner's Manual of Psychoeducational Profile (3rd ed.). Austin, TX: Pro-ed Incorporation. https://doi.org/10.1037/t52601-000

Schopler, E., Reichler, R., \& DeVellis, R. (1980). Toward Objective Classification of Childhood Autism: Childhood Autism Rating Scale (CARS). Journal of Autism and Developmental Disorders, 10, 91-103. https://doi.org/10.1007/BF02408436

Schopler, E., Reichler, R., \& Rochen Renner, B. (1988). The Childhood Autism Rating Scale. Western Psychological Services.

Warner, G., Moss, J., Smith, P., \& Howlin, P. (2014) Autism Characteristics and Behavioral Disturbances in -500 Children with Down's Syndrome in England and Wales. Autism Research, 7, 433-441. https://doi.org/10.1002/aur.1371 